\title{
Characterization of kinesin-like proteins in silkworm posterior silkgland cells
}

Qiao Wang, Junlin Teng, Birong Shen, Wei Zhang, Yige Guo, Xiaolei Su, Chuanxi Zhang, Albert CH Yu, Jianguo Chen

Cell Research (2010) 20:859. doi:10.1038/cr.2010.91; published online 1 July 2010

Correction to: Cell Research (2010) 20:713-727. doi:10.1038/cr.2010.47; published online 6 April 2010

Due to a production error, a mistake has been introduced in the doi number of this paper in the print version. The corrected doi number is as follows:

Cell Research (2010) 20:713-727. doi:10.1038/cr.2010.47; published online 6 April 2010 